\title{
Risk factors for adverse perinatal outcomes depend on smoking status, a study from a developing country
}

\author{
Shereen Hamadneh ${ }^{1}$ and Jehan Hamadneh ${ }^{2}$
}

\author{
${ }^{1} \mathrm{Al}$ al-Bayt University \\ ${ }^{2}$ Affiliation not available
}

September 1, 2021

\begin{abstract}
Background: This study aimed to investigate the risk factors for smoking and negative perinatal outcomes among Jordanian women about smoking status. Methods: A case-control study was conducted among singleton full-term pregnant women who gave birth at the main hospital in Jordan in June 2020. They were divided into three groups according to their smoking status (active, passive and non-smokers) and were interviewed using a semi-structured questionnaire, including demographic information, current pregnancy history, perinatal and neonatal outcomes. The study investigated the effect of smoking status on both independent and dependent variables. Results: Our study revealed that low-level maternal education $(\mathrm{OR}=25.38)$, unemployed maternal status $(\mathrm{OR}=2.67)$, the absence of following up during pregnancy $(\mathrm{OR}=5.8)$, smoking husband were risk factors for smoke exposure among pregnant women. The risk for cesarean section was increased in nulliparous smoking women $(\mathrm{OR}=9.0)$, those with low family monthly income $(\mathrm{OR}=7.8)$, and those who did not get any information about the hazard effect of smoking ( $\mathrm{OR}=4.38)$, as well as in unemployed passive smoking women $(\mathrm{OR}=6.25)$. Parity of more than one has raised the risk of NICU admission inactive smoking women $(\mathrm{OR}=10.38)$. This risk was also increased in active and passive women with a lower level of education $(\mathrm{OR}=186.33$ and $\mathrm{OR}=17.5)$, respectively, as well as inactive smoking women with low family monthly income $(\mathrm{OR}=4.11)$. Conclusions: Appropriate preventive strategies should focus on modifiable risk factors for smoking during pregnancy.
\end{abstract}

\section{Hosted file}

[3]_Smoking 4 outcomes OR 29.06.2021.edited (1).docx available at https://authorea.com/users/ 369061/articles/535987-risk-factors-for-adverse-perinatal-outcomes-depend-on-smokingstatus-a-study-from-a-developing-country 